\title{
Resolution limits of laser spectroscopic absorption measurements with hollow glass waveguides
}

\author{
Jia Chen, ${ }^{1,2, *}$ Andreas Hangauer, ${ }^{1,2}$ Rainer Strzoda, ${ }^{1}$ and Markus Christian Amann ${ }^{2}$ \\ ${ }^{1}$ Corporate Research and Technologies, Siemens AG, Otto-Hahn-Ring 6, D-80200 Munich, Germany \\ ${ }^{2}$ Walter Schottky Institut, Technische Universität München, Am Coulombwall 3, D-85748 Garching, Germany \\ ${ }^{*}$ Corresponding author: jia.chen@wsi.tum.de
}

Received 1 June 2010; accepted 22 June 2010;

posted 13 August 2010 (Doc. ID 129256); published 22 September 2010

\begin{abstract}
In this paper, resolution limits of laser spectroscopy absorption measurements with hollow capillary fibers are investigated. Furthermore, a concept of sensitive near-infrared sensing utilizing hollow fiber directly coupled with vertical-cavity surface-emitting lasers is developed. By performing wavelength modulation spectroscopy, the smallest absorbance that can be detected by the fiber sensor was determined to be $10^{-4}$, limited by a random modulation of the fiber transmission function (modal noise). By mechanically vibrating the fiber, a sensor resolution of $10^{-5}$ in absorbance is achieved. Because the random modulation on the fiber transmission function limits the detection sensitivity, its physical reasons are analyzed. One contribution is found to be the partial integration of the far field, and the amplitude of the spectral features is inversely proportional to the square root of the integrated speckle points number. Therefore, careful design of the fiber-detector outcoupling is necessary. It turned out that incoupling alignment is not of much influence with respect to the spectral background. The residual spectral background is caused by mode-dependent effects and can be lowered by vibrating the fiber mechanically. (C) 2010 Optical Society of America

OCIS codes: $\quad 300.6360,060.2370$.
\end{abstract}

\section{Introduction}

Tunable diode laser spectroscopy based gas sensors using fibers as both gas "pipe" and optical waveguide provides a much longer path length, compared to conventional single-reflective gas cells [1]. Furthermore, it greatly reduces the sample volume requirement compared to conventional sample cells, e.g., Herriott cell [2] and White cell [3].

One type of gas-permeable/gas-filled fiber is the hollow capillary fiber/hollow glass waveguide (HGW) [4], which was originally developed for transmitting $\overline{\mathrm{CO}}_{2}$ laser power at the $10.6 \mu \mathrm{m}$ range [5] and YAG laser power at $2.94 \mu \mathrm{m}$ [6]. It is a highly multimode fiber, i.e., there is a multitude of different paths for the light propagating in the fiber. Because many physical effects, such as backscattering at the end of the fiber, absorption in the coating, or scattering in-

0003-6935/10/285254-08\$15.00/0

(C) 2010 Optical Society of America side the fiber are mode-dependent, the superposition of the many fiber modes at the end of the fiber may result in random modulation of the fiber transmission function. This means that with increasing wavelength but constant light intensity at the fiber input, intensity fluctuations/modal noise appear at the fiber output. If these spectral features exhibit a periodicity similar to the linewidth of the absorbing species (FWHM, 2-4 GHz at atmospheric pressure) and exceed other noise, e.g., detector noise, they will become the limitation of the detection sensitivity of the sensor. Detectors for the near-infrared range (NIR) give noise limits much lower than $10^{-5}$ with a typical photodetector power of more than $1 \mu \mathrm{W}$ and $1 \mathrm{~Hz}$ in bandwidth. The single mode fiber has modal noise on the transmission function of the order of approximately $10^{-4}$ due to interfering with the other polarization mode. The modal noise will increase if the fiber is stressed or tightly wound. Methods for compensating the unwanted distortions, including the 
fiber background, exist to achieve a resolution of absorbance in the $5 \times 10^{-6}$ range. However, a reference channel is needed $[7,8]$.

Several gas/liquid sensing experiments with hollow glass waveguides have been reported: with quantum cascade lasers for midinfrared sensing using coupling elements such as objective or off-axis parabolic mirrors $[9,10]$; or experiments focusing on the concentration resolution achievable for ethylene at $10 \mu \mathrm{m}[11,12]$. However, two relevant aspects are missing in the literature: no characterization of the sensor sensitivity limit with respect to the smallest detectable absorbance was done, and no NIR laser based measurement was carried out so far. In this paper, the smallest absorbance resolvable with fibers in the NIR $(0.8-2.5 \mu \mathrm{m})$ is investigated. The concentration resolution $c_{\text {res }}$ (mixing ratio) can then be calculated with the known fiber length $l$ and absorption coefficient $\alpha$ (unit: $1 / \mathrm{m}$ ), i.e., $c_{\text {res }}=\frac{a_{\text {res }}}{l \alpha}$. For the investigations, the hollow fiber is directly coupled to vertical-cavity surface-emitting lasers (VCSELs) [13]. VCSELs cover the wavelength range from 763 to $2365 \mathrm{~nm}$ [13-15], and the small divergence of VCSEL emission (FWHM: 10 to $20^{\circ}$ ) makes them attractive sources for direct coupling with the hollow fiber. Fiber sensors utilizing hollow capillary fibers and VCSELs are a compact and cost-efficient solution for gas sensing, and this concept shall be proved in this paper.

\section{Fundamentals}

In this section, the properties of hollow capillary fibers are introduced. Furthermore, wavelength modulation spectroscopy (WMS) and its capability to attenuate the interference on the gas absorption spectrum is elucidated.

\section{A. Fiber Properties}

The hollow capillary fiber is an air- or gas-filled hollow waveguide, where the light is guided in the core by reflections on the AgI/Ag coating on the inner surface of the fiber. These fibers, with diameters from 200 to $1000 \mu \mathrm{m}$, as well as lengths up to $13 \mathrm{~m}$, are commercially available [4]. For our investigations, we used a fiber with a $75 \overline{0} \mu \mathrm{m}$ inner diameter (Polymicro, HWEA7501200). Its structure is shown in Fig. $1[5,16]$. The AgI coating has an optimized thickness of $0.3 \mu \mathrm{m}$ for a minimal loss at a wavelength of $2.94 \mu \mathrm{m}$ [4]. The fiber shows an attenuation of 2 to $4 \mathrm{~dB} / \mathrm{m}$ at a 2 to $3 \mu \mathrm{m}$ wavelength. This hollow glass structure has a very smooth inner surface compared to other hollow structures [4]. Its volume per optical interaction length is $400 \bar{\mu} \mathrm{Liter} / \mathrm{m}$, which is very small compared to conventional sample cells, e.g., a Herriott cell with a volume of $0.27 \mathrm{~L}$ for an optical path length of $36 \mathrm{~m}$ [17].

\section{B. Wavelength Modulation Spectroscopy: Interference Attenuation}

For the recording of the gas absorption spectra, we utilize WMS [18], which is an established method

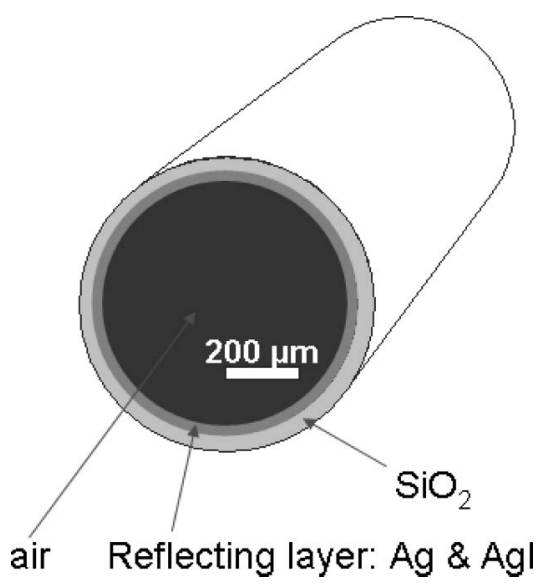

Fig. 1. Schematic figure of the hollow capillary waveguide with an inner diameter of $750 \mu \mathrm{m}$. It has a silica cladding and a thin metallic film of $\mathrm{Ag}$ and a single dielectric film of AgI, both deposited inside the cladding [4].

for sensitive absorbance measurements. Its advantages are efficient noise suppression and removal of the laser amplitude modulation [19] in the measured data. The measured signal is not the transmission spectrum $I / I_{0}$, but the second harmonic spectrum $I_{2} / I_{0}$, where $I$ is the intensity at the output of the fiber and $I_{0}$ is the averaged intensity at the output of the fiber with spectral features removed. Detection of the second harmonic yields the second derivative, or more exactly, a bandpass filtering of the transmission spectrum [20]. The relationship between $I_{2} / I_{0}$ and $I / I_{0}$ is shown in Fig. 2. The Fourier transform of the detected second harmonic is the product of the Fourier transform of the detected transmission spectrum and a 2nd order Bessel function [20]. If additional low pass filtering in the lock-in amplifier (SRS, Model SR830 [21]) is taken into account, the following formula is obtained:

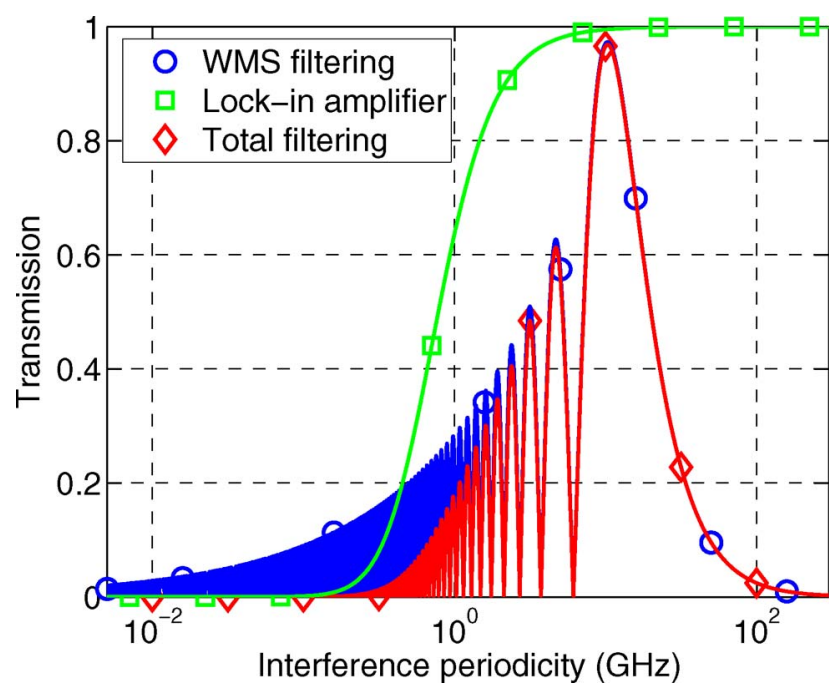

Fig. 2. (Color online) Filtering achieved by WMS with a modulation amplitude of $5 \mathrm{GHz}$ (blue curve with circles) and by the filter inside the lock-in amplifier in a typical system (green curve with rectangles). The product of both (red curve with diamonds) gives the relationship between the measured second harmonic and the transmission spectrum in the frequency domain. 


$$
\begin{aligned}
\mathcal{F}\left\{\frac{I_{2}(\lambda)}{I_{0}(\lambda)}, k\right\}= & \mathcal{F}\left\{\frac{I(\lambda)}{I_{0}(\lambda)}, k\right\} \cdot(-2) J_{2}(2 \pi \Delta \lambda k) \\
& \cdot \frac{1}{(1+i 2 \pi k \tau \beta)^{n}},
\end{aligned}
$$

where $\lambda$ and $k$ denote wavelength and wavenumber, and $\Delta \lambda$ denotes modulation amplitude in wavelength. $\lambda$ and $k$ are conjugate variables of the Fourier transform $\mathcal{F}: \mathcal{F}(f(\lambda), k)=\int_{-\infty}^{\infty} f(\lambda) \exp (-2 \pi i \lambda k) \mathrm{d} \lambda$.

The lock-in filter is an $n$th order low pass with its cutoff frequency depending on the lock-in time constant $\tau[\mathrm{s}]$ and the spectrum sweep rate $\beta[\mathrm{nm} / \mathrm{s}]$. The typical values are $\tau=1 \mathrm{~ms}, \beta=\Delta \lambda 16 / \mathrm{s}$, and $n=$ $4(24 \mathrm{~dB} /$ oct $)$. The spectral features on the transmission spectrum are partially attenuated by detecting the second harmonic spectrum. Therefore, the interference on the second harmonic is relatively narrowband even if broadband interferences on the transmission spectrum exist.

Usually, the modulation amplitude $\Delta \lambda$ is chosen to $2.2[18,22,23]$ or 3 times of the HWHM (half-width at half-maximum) $[23,24]$, so that the peak value or the peak-to-peak value of the second harmonic spectrum is maximum. In the experiments, the modulation amplitude was set to $5 \mathrm{GHz}$ to obtain the maximum gas absorption signal (HWHM at ambient conditions of $1-2 \mathrm{GHz}$ ). According to Fig. $\underline{2}$, the least attenuated interference periodicity is therefore $10 \mathrm{GHz}$.

\section{Measurements of Fiber Spectral Background}

In this section, we show the characterization results of the relative intensity fluctuation at the second harmonic spectrum $I_{2} / I_{0}$ of $\mathrm{HGW}$, which is the detection limitation of the fiber sensor.

\section{A. Experimental Setup: Direct Coupling of Hollow Capillary Fiber and VCSELS}

The experimental setup of a directly coupled VCSEL to hollow capillary fiber is shown in Fig. 3. We used VCSELs emitting at 1.6 and $2.3 \mu \mathrm{m}$ wavelength, where water vapor and methane gas have strong absorption lines. Direct coupling is realized by placing the fiber directly in front of the laser chip at a distance $\leq 1 \mathrm{~mm}$. The VCSEL has an aperture diameter of approximately $5 \mu \mathrm{m}$ and a small divergence $\left(10-20^{\circ} \mathrm{FWHM}\right)$, therefore the direct incoupling is straightforward and exhibits low loss. Neither lenses nor mirrors were used in this experimental setup. A slightly tilted Ge photodiode (3 $\mathrm{mm}$ diameter) and an

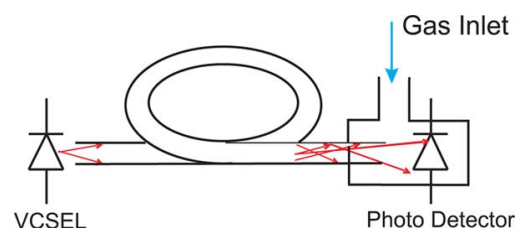

Fig. 3. (Color online) Direct coupling of the VCSEL and the HGW. The fiber is filled with gas through the gas inlet. The couple element is sealed to the photodetector, gas inlet, and fiber end. For the spectral background measurement, the fiber is filled with nitrogen.
InGaAs photodiode ( $1 \mathrm{~mm}$ diameter) are used for detection at 1.6 and $2.3 \mu \mathrm{m}$, respectively.

\section{B. Characterization of Sensing Limitation}

The absorbance resolution of a standard singlereflection cell is of the order of $10^{-5}$ [Fig. 4(a)]. The smallest absorption that can be detected with the $\mathrm{HGW}$ is characterized by measuring the second harmonic spectrum of a nitrogen-filled fiber, which shows a tiny structure of the order of $10^{-4}$, i.e., variations of the order of $10^{-4}$ exist on $I_{2} / I_{0}$ [Fig. $4(\mathrm{~b})$ ]. The standard deviation of the variation $\sigma\left(I_{2}\right) / I_{0}$ is $6 \times 10^{-5}$. This structure is not a single sinusoidal but consists of many frequency components with a most dominant periodicity of approximately $10 \mathrm{GHz}$ ( $=0.1 \mathrm{~nm} @ 1.68 \mu \mathrm{m})$, which corresponds to the interference periodicity least attenuated due to second harmonic detection (see Fig. 2). A structure with $0.1 \mathrm{~nm}$ periodicity can be produced by a $1 \mathrm{~cm}$ long etalon. However, there are no parallel surfaces with a distance in this range that could form an etalon in our measurement system, excluding other reasons, except for the fiber itself causing the background. The possibility of reflections from the first section of the fiber striking back to the laser is also excluded, because the interference periodicity does not change with distance between fiber and laser. Spectral features of the order of $10^{-4}$ with a most dominant periodicity of $10 \mathrm{GHz}$ are also observed at $2.36 \mu \mathrm{m}$.

\section{Analysis of Fiber Spectral Background}

The theoretical detection limit given by the detector noise in the NIR $(\lambda \leq 2.5 \mu \mathrm{m})$ is of the order of $10^{-7}$ even if the photodetector current is as low as $1 \mu \mathrm{A}$ and the $1 \mathrm{~Hz}$ detection bandwidth is employed. A $10^{-5}$ shot noise is realized if power in only the $\mathrm{nW}$ range is arriving at the photodetector, corresponding to the attenuation of more than $15 \mathrm{~m}$ of hollow fiber (using a conservative attenuation value of $4 \mathrm{~dB} / \mathrm{m}$ and laser power of $1 \mathrm{~mW}$ ). Therefore, spectral background/spectral features on the fiber transmission function limit the detection sensitivity. It is

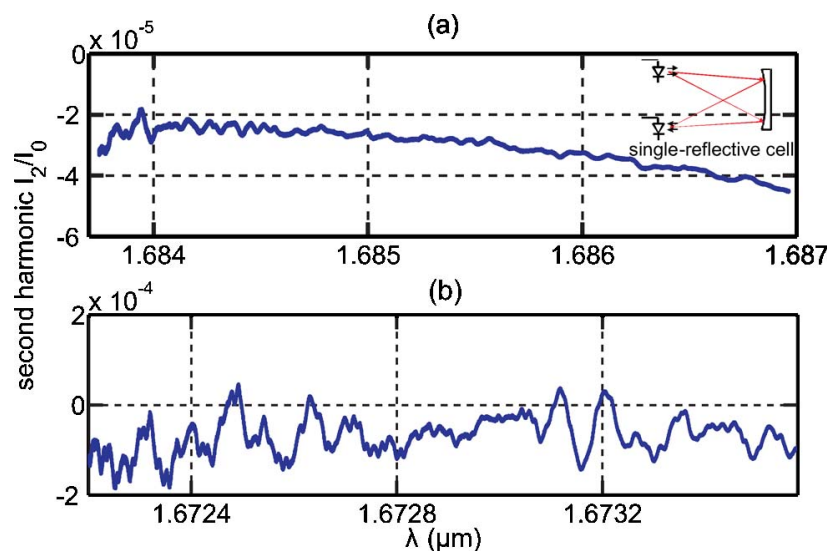

Fig. 4. (Color online) Spectral background of (a) a standard spherical mirror based single-reflection cell including laser background (peak to peak $10^{-5}$ ) and (b) a HGW fiber (peak to peak $2 \times 10^{-4}$, standard deviation $\sigma\left(I_{2}\right) / I_{0}$ is $6 \times 10^{-5}$ ). 
important to understand the reason for it and to reduce it, if possible.

\section{A. Far-Field Observations and Analysis}

It is assumed that the spectral features result from the multimode character of the fiber. Therefore, it is essential to know how many fiber modes are excited, and also the distribution of these modes at the fiber end. For the following cases, far fields were measured with an InSb-based camera without objective (Cedip, Titanum):

- straight fiber $(20 \mathrm{~cm})$ with a centrally coupled VCSEL [Fig. 5(a)].

- straight fiber $(20 \mathrm{~cm})$ with a noncentrally coupled VCSEL with $100 \mu \mathrm{m}$ lateral displacement [Fig. 5(b)].

- bent fiber (3 m) with a bend radius of approximately $15 \mathrm{~cm}$ [Fig. $5(\mathrm{c})]$.

The distance between fiber end to camera is approximately $3 \mathrm{~cm}$.

\section{Number of Excited Modes}

In the case of the straight fiber with a center coupled VCSEL, the far field shows a ring structure, which indicates that only circularly symmetric modes are excited, i.e., only modes with a Bessel function of order 0. For a straight fiber with laterally displaced coupling and for a bent fiber, a speckle pattern is obtained. It is a spatial intensity pattern, i.e., a spatially varying superposition of many modesincluding higher order azimuthal modes $[25,26]$. According to Freude [26], the maximum number of excited modes in a multimode fiber is determined by

$$
M=\left(\frac{2 \pi}{\lambda} \tan (\theta) a\right)^{2},
$$

with $a$ and $\lambda$ denoting the inner radius of the hollow waveguide and the wavelength of the emitted light, respectively. According to Fig. 5(c), we determined the acceptance angle of the fiber $\theta$ to be approximately $6^{\circ}$ (HWHM), which gives a maximum of 10,000 modes propagating in the bent fiber and coupled out of it. Modes corresponding to angles much higher than the acceptance angle have very high losses, and thus, light in these modes does not propagate over the length of the fiber.

\section{Statistic of Modes Distribution}

The spatial intensity distribution of speckle pattern caused by diffuse reflection is theoretically an exponential distribution [27]:

$$
P(I)=\exp (-I /\langle I\rangle),
$$

with $\langle I\rangle$ being the spatial mean value of the intensity and $P$ is the probability density function. After diffuse reflections, different light modes are uncorrelated to each other and the E-field strength of each light mode is orthogonal to each other, which results in the Gaussian-distributed sum E-field. Intensity is the sum of the squared real and imaginary parts of the E-field, and therefore, its distribution is an exponential distribution. The spatial intensity distribution of the recorded far field of a $3 \mathrm{~m}$ bent fiber is plotted in Fig. 6 (red solid curve), which clearly shows an exponential behavior (blue dashed curve in Fig. 6 ). This gives an indication that the different fiber modes in the bent hollow waveguide are uncorrelated to each other and add with statistical phase and amplitude. This knowledge could be deployed for the simulation of the fiber far field. The spatial intensity distribution of the far field of a straight fiber with noncentral incoupling [Fig. 5(b)] was also found to be an exponential distribution.

\section{Far Field During Wavelength Scanning}

If the integrated speckle intensity stays the same during the wavelength scan, the fiber transmission function should be smooth, i.e., $\sigma\left(I_{2}\right) / I_{0}=0$. During wavelength tuning, the fiber mode compositions change, and therefore, also their constructive or destructive superposition at a certain spot of the photodetector, i.e., the speckle pattern changes. The recorded speckle pattern changed slightly when the laser wavelength was tuned for several $\mathrm{nm}$. The change at the perimeter is clearly observable,

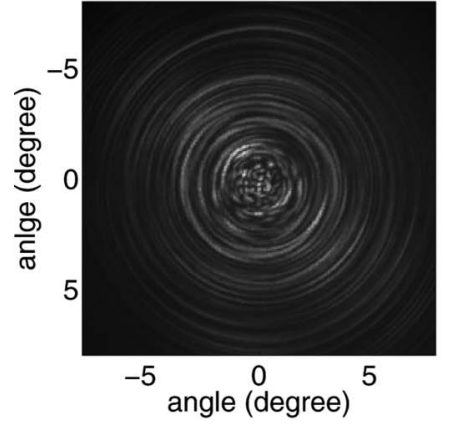

(a)

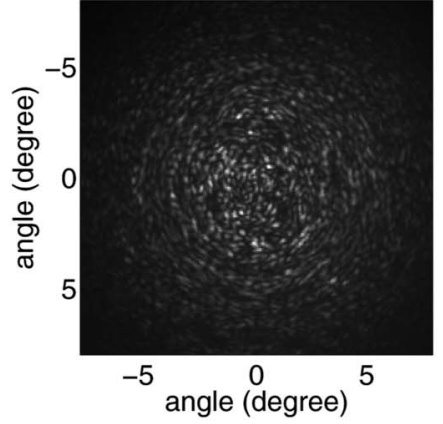

(b)

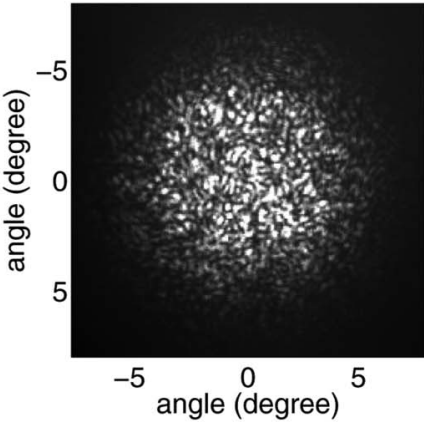

(c)

Fig. 5. Far field of hollow fibers directly coupled with VCSELs. 


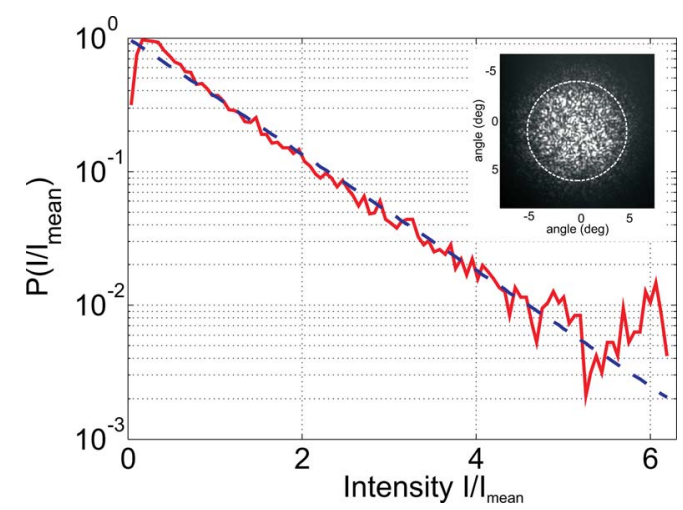

Fig. 6. (Color online) Spatial intensity distribution of the observed far field of a $3 \mathrm{~m}$ bent fiber (red solid curve) and the theoretical spatial intensity distribution of a speckle pattern (blue dashed line). The area of far field being evaluated is indicated by a circle in the inset. The $y$ axis is in logarithmic scale. The noise at high intensity is because of the small number of measurement data.

whereas in the center it is hardly noticeable. The possible reason is the following: speckle points at the perimeter are caused by interfering light modes with very different propagation constants, leading to etalon fringes with small frequency spectral range (FSR). In the middle, the FSR of etalon fringes are relatively large-much larger than the wavelength range being tuned.

According to the law of energy conservation, the spectral features can only be caused if the photodetector does not collect all the light coming from the fiber end, and/or mode-dependent losses exist. Therefore, the next step is to investigate the contribution of different incoupling/outcoupling to the fiber spectral background, i.e., the dependence on the radial incoupling position and dependence on partial integration of the fiber far field.

\section{B. Spectral Background and Incoupling}

In the experiments shown in this section and Subsection 4.C, we used a slightly tilted Ge photodiode ( $3 \mathrm{~mm}$ diameter) and a $1.68 \mu \mathrm{m}$ VCSEL tuned to a wavelength range, where absorptions due to ambient gases in the fiber with a $3 \mathrm{~m}$ optical path length are less than $2 \times 10^{-6}$. Therefore, for the spectral background characterization, there is no need to fill the fiber with nitrogen.

We investigated the dependency of spectral features to the incoupling using the experimental setup shown in Fig. 7, with the photodetector directly coupled to the end of the $20 \mathrm{~cm}$ straight fiber. The lateral displacement of the VCSEL from the fiber center

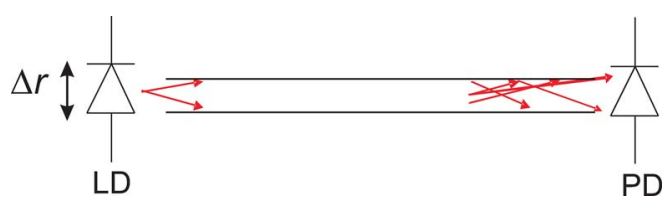

Fig. 7. (Color online) Incoupling experiment with a $20 \mathrm{~cm}$ straight fiber, where the lateral displacement between the VCSEL and the fiber center $\Delta r$ is varied.

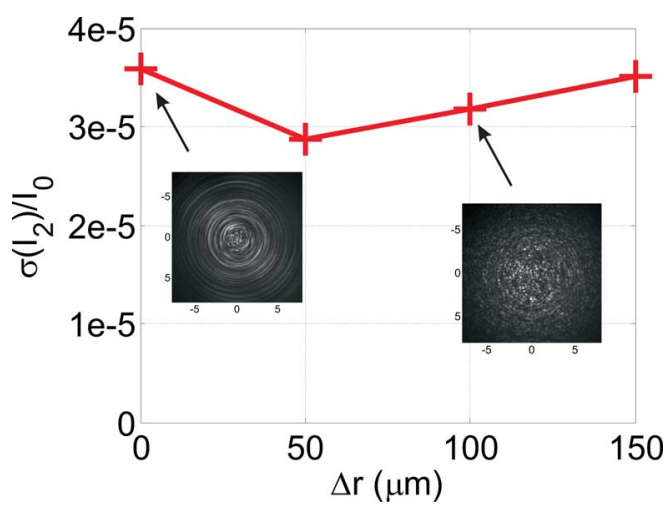

Fig. 8. (Color online) $\sigma\left(I_{2}\right) / I_{0}$ versus $\Delta r$ (lateral laser displacement) in $\mu \mathrm{m}$. The insets show the fiber far fields for $\Delta r=0 \mu \mathrm{m}$ and $100 \mu \mathrm{m}$.

$\Delta r$ is varied from 0 to $150 \mu \mathrm{m}$ in $50 \mu \mathrm{m}$ steps. $\Delta r=0$ gives a ring structure as the far field, and the resulting spectral feature has a similar period but slightly higher standard deviation, as in the case for sideways coupling $(\Delta r \neq 0)$, in which a speckle pattern is observed (Fig. 8). The far field of a bent fiber is always a speckle pattern independent of incoupling. Therefore, with regard to the fiber spectral background, precise incoupling is not an issue for gas sensing with hollow capillary fibers.

\section{Spectral Background and Outcoupling}

To determine the influence of partial collection of the light coming from the fiber on the fiber background, the distance between the fiber end and the photodiode $\Delta z$ is varied between $1 \mathrm{~mm}$ and $12 \mathrm{~cm}$ (Fig. 9). The standard deviation of the spectral features against $\Delta z$ is shown in Fig. $10 . \sigma\left(I_{2}\right) / I_{0}$ increases linearly with $\Delta z$, indicating its antiproportional dependency on the diameter of the integrated far-field area and thus the square root of $N$ (the number of the integrated speckles), i.e., $\sigma\left(I_{2}\right) / I_{0} \propto 1 / \sqrt{N}$. Below a certain distance, however, the linear decrease of the fiber background level comes to a threshold because $N$ stays constant. These residual spectral background are caused by mode-dependent losses. These constitute the following:

- backscattering at the end of the fiber;

- incoupling losses: backscattering at the input of the fiber;

- responsitivity of the photodiode. The responsitivity of the photodiode depends on the incident angle of the photons, which is mode-dependent.

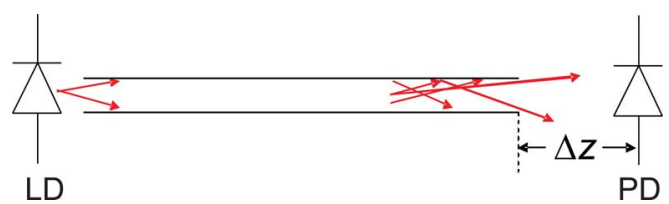

Fig. 9. (Color online) Outcoupling experiment with $20 \mathrm{~cm}$ straight fiber: the area of the integrated far field by photodiode is varied by changing $\Delta z$. 


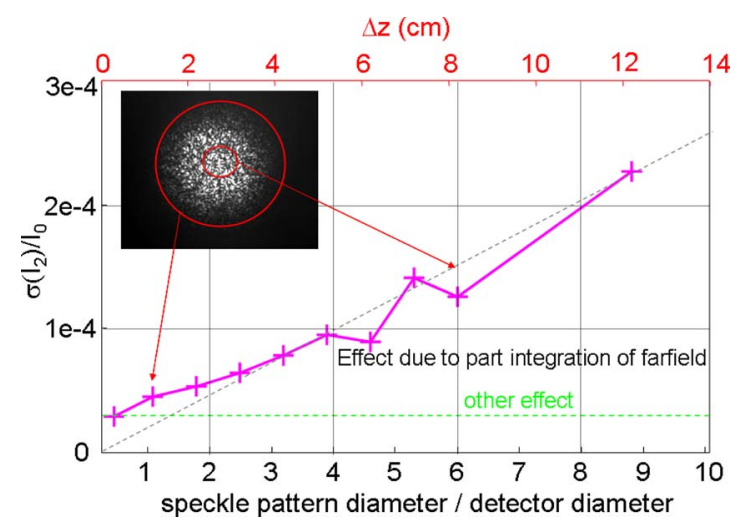

Fig. 10. (Color online) $\sigma\left(I_{2}\right) / I_{0}$ against the diameter ratio between the detector and the integrated speckle pattern, which is varied by changing $\Delta z$. A $20 \mathrm{~cm}$ straight fiber was investigated. At $\Delta z=1 \mathrm{~cm}$, the speckle pattern diameter is approximately equal to the detector diameter and the background level goes to a threshold. These residual spectral backgrounds are caused by modedependent losses.

The above effects are independent on fiber length and bent situation, whereas the following ones depend on these parameters:

- scattering in the fiber because of the inhomogeneity of the fiber or particles in the fiber;

- absorption in the coating (Ag/AgI), which increases when the fiber is bent.

To quantify influences caused by the bendingspecific loss mechanism, the previous experiment is repeated for the bent fiber (experimental setup in Fig. 11). Additionally, vibration is applied to reduce the influence of all possible mode-dependent effects on the fiber transmission. By vibration, the speckle pattern changes over time, which effectively gives a larger number of $N$ integrated by the photodiode. The results are shown in Fig. 12. The value at the origin for the $3 \mathrm{~m}$ bent fiber (curve marked by diamonds) gives the upper limit of the mode-dependent effects. This value is lower for the $20 \mathrm{~cm}$ straight fiber, because bending losses do not exist and the lower scattering effect is inside the shorter fiber (curve marked by crosses). Furthermore, there seems to be a constant improvement factor achievable by fiber vibration compared to a nonvibrating system (roughly

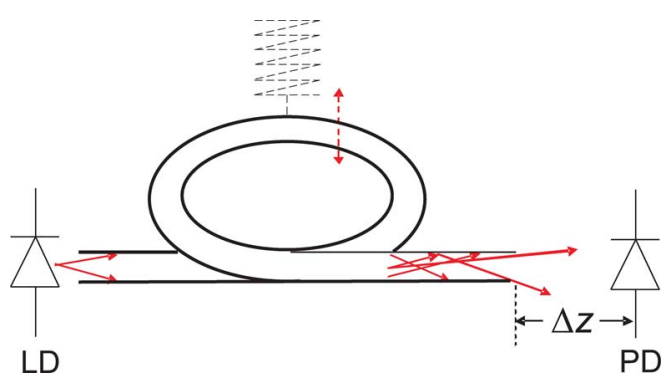

Fig. 11. (Color online) Outcoupling experiment with a $3 \mathrm{~m}$ bent fiber: the integrated far-field area is varied by changing $\Delta z$. The experiments were done for stationary and mechanically vibrated fibers.

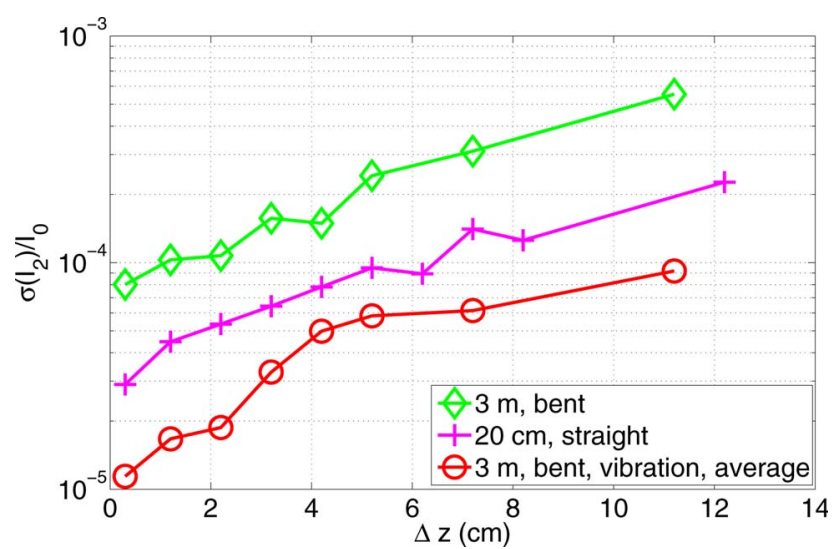

Fig. 12. (Color online) Standard deviation of the interference structure $\sigma\left(I_{2}\right) / I_{0}$ against $\Delta z$ : a $3 \mathrm{~m}$ bent fiber is investigated in stationary (diamond) and vibration condition (circle). For comparison, the result of a $20 \mathrm{~cm}$ straight fiber is also indicated by a cross (Fig. 10). The $y$-axis is in logarithmic scale.

constant distance between the curve marked by circles and diamonds on a logarithmic scale). By vibrating the fiber faster compared to the speed of wavelength tuning, the speckle pattern varies strongly at one wavelength, which corresponds to different distributions of fiber modes with respect to the amplitude and phase, giving different speckle pattern realizations as far field. The averaged intensity of different speckle pattern realizations is rather wavelength independent and therefore results in a smoother fiber transmission function. As a result, a spectral background as low as $1 \times 10^{-5}$ is achieved by vibrating the fiber and placing the photodiode very close to the fiber end.

\section{Sensor Solution and Performance}

According to the above sections, now the actions that need to be undertaken to realize a high resolution fiber sensor are obvious: use a large photodiode placed as close as possible to the fiber end to integrate the whole speckle pattern, and vibrate the fiber to realize many speckle realizations. These are then averaged at each wavelength, so the residual spectral features caused by the wavelength-dependent loss mechanism are smoothed out. In our experiments, the vibration is a frequency sweep from 10 to $100 \mathrm{~Hz}$ with a repetition rate of $10 \mathrm{~Hz}$, and the vibration amplitude is several hundred $\mu \mathrm{m}$. The wavelength sweep is

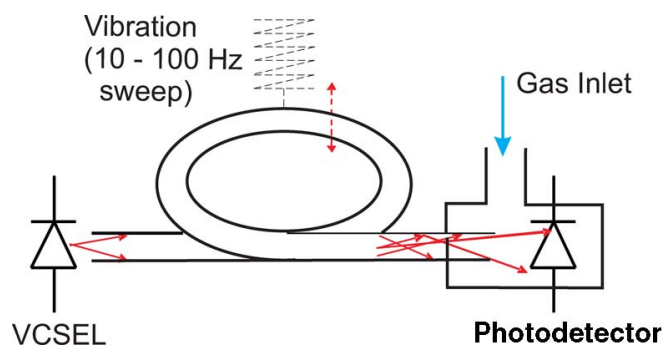

Fig. 13. (Color online) Schematic figure of the fiber sensor: the $3 \mathrm{~m}$ hollow capillary fiber is directly coupled to the VCSELs and vibrated mechanically. 
(a)

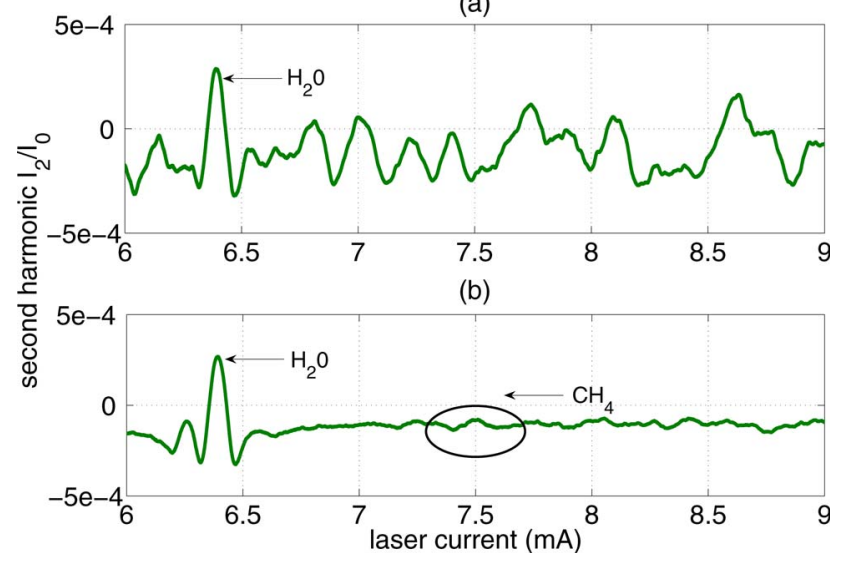

Fig. 14. (Color online) (a) Second harmonic spectrum without fiber vibration at $2365 \mathrm{~nm}$. (b) Second harmonic spectrum at $2365 \mathrm{~nm}$ with additional mechanical vibration of the fiber. The laser wavelength is tuned with current, which is shown on the $x$ axis. The wavelength tuning range is approximately $3 \mathrm{~nm}$

accomplished every second. The modulation frequency for WMS is $6 \mathrm{kHz}$. By less than $40 \mathrm{~s}$ averaging, the spectral background exhibits 10 times lower noise than without vibration. As a result, the $1.5 \mathrm{ppm}$ background level of methane absorption in the ambient air could be detected at $2.365 \mu \mathrm{m}$ with a $3 \mathrm{~m}$ hollow capillary fiber [Fig. 14(b)]. This corresponds to an absorption resolution in the range of $10^{-5}$. During revision of this paper the authors were able to improve the required averaging time from $40 \mathrm{~s}$ to $100 \mathrm{~ms}$ [28].

\section{Conclusion}

In this paper, resolution limits of laser spectroscopy absorption measurements with hollow capillary fiber have been investigated, and a concept of sensitive and compact near-infrared sensing with hollow capillary fibers directly coupled with VCSELs was developed. Employing wavelength modulation spectroscopy, the fiber transmission function has a random modulation of the order of $10^{-4}$. With additional mechanical vibration of the fiber, a spectral resolution of $10^{-5}$ is achieved with the fiber sensor. This is of the order of what is also reachable with a typical single-reflection cell [1]. Therefore, the fiber sensor has a better concentration resolution because of the typically much longer optical path length.

Because the random modulation on the fiber transmission function limits the detection sensitivity, we analyzed the physical reason for it. The fiber far field is generally a speckle pattern, and its spatial intensity is exponentially distributed. This gives an indication that the 10,000 fiber modes are independently distributed with respect to phase and amplitude. One contribution for the spectral feature is found to be the partial integration of the far field, and the amplitude of the spectral features is inversely proportional to the square root of the integrated speckle points number. Therefore, a setup with a large photodiode and its distance to fiber end as short as possible is believed to be nonsusceptible to the occurrence of interference fringes. The incoupling is not critical with respect to the fiber background-displacements from 0 to $150 \mu \mathrm{m}$ from the central coupling have no influence on the amplitude of the spectral background. The residual spectral background is caused by mode-dependent effects such as backscattering and absorption and can be lowered by averaging many speckle pattern realizations at one wavelength-practically realized by vibrating the fiber mechanically.

Jia Chen thanks Ralf Meyer for the valuable discussions, and the authors acknowledge the Federal Ministry of Education and Research of Germany for financial support (Project "Nose," 13N877).

\section{References}

1. J. Chen, A. Hangauer, R. Strzoda, and M.-C. Amann, "Laser spectroscopic oxygen sensor using diffuse reflector-based optical cell and advanced signal processing," Appl. Phys. B 100, 417-425 (2010).

2. D. Herriott, H. Kogelnik, and R. Kompfner, "Off-axis paths in spherical mirror interferometers," Appl. Opt. 3, 523-526 (1964).

3. J. U. White, "Long paths of large aperture," J. Opt. Soc. Am. 32, 285 (1942)

4. J. A. Harrington, "A review of IR transmitting, hollow waveguides," Fiber Integr. Opt. 19, 211-217 (2000).

5. J. A. Harrington, C. Rabii, and D. Gibson, "Transmission properties of hollow glass waveguides for the delivery of $\mathrm{CO}_{2}$ surgical laser power," IEEE J. Sel. Top. Quantum Electron. 5, 948-953 (1999).

6. Y. Matsuura, T. Abel, and J. A. Harrington, "Optical properties of small-bore hollow glass waveguides," Appl. Opt. 34, 68426847 (1995).

7. S. Lundqvist and P.-A. Thorsen, "Wavelength modulation spectroscopy method and system," U.S. patent 7,193,718 (20 March 2007).

8. S. Lundqvist and P. Kluczynski, "Method for improving the sensitivity in a fiber coupled diode laser spectrometer by selective predistortion," in Book of Abstracts: Field Laser Applications in Industry and Research (FLAIR) (2007), p. 39, http://www.inoa.it/flair.

9. S. Hartwig and A. Lambrecht, "Characterization of hollow fibres for compact infrared gas measurement cells," Tech. Mess. 75, 555-564 (2008).

10. C. Young, S.-S. Kim, Y. Luzinova, M. Weida, D. Arnone, E. Takeuchi, T. Day, and B. Mizaikoff, "External cavity widely tunable quantum cascade laser based hollow waveguide gas sensors for multianalyte detection," Sens. Actuators. B 140, 24-28 (2009).

11. C. A. Worrell and N. A. Gallen, "Trace-level detection of gases and vapours with mid-infrared hollow waveguides," J. Phys. D 30, 1984-1995 (1997).

12. L. Hvozdara, S. Gianordoli, G. Strasser, W. Schrenk, K Unterrainer, E. Gornik, C. S. S. S. Murthy, M. Kraft, V. Pustogow, B. Mizaikoff, A. Inberg, and N. Croitoru, "Spectroscopy in the gas phase with GaAs/AlGaAs quantum-cascade lasers," Appl. Opt. 39, 6926-6930 (2000).

13. M.-C. Amann and M. Ortsiefer, "Long-wavelength $(\lambda \geq 1.3 \mu \mathrm{m})$ InGaAlAs-InP vertical-cavity surface-emitting lasers for applications in optical communication and sensing," Phys. Status Solidi A 203, 3538-3544 (2006).

14. M. Grabherr, D. Wiedenmann, R. Jaeger, and R. King, "Fabrication and performance of tunable single-mode VCSELs 
emitting in the 750 to $1000 \mathrm{~nm}$ range," Proc. SPIE 5737, 120 128 (2005)

15. A. Bachmann, T. Lim, K. Kashani-Shirazi, O. Dier, C. Lauer, and M.-C. Amann, "Continuous-wave operation of electrically pumped GaSb-based vertical cavity surface emitting laser at $2.3 \mu \mathrm{m}$," Electron. Lett. 44, 202-203 (2008).

16. R. K. Nubling and J. A. Harrington, "Launch conditions and mode coupling in hollow-glass waveguides," Opt. Eng. 37, 2454-2458 (1998).

17. J. B. McManus, "Paraxial matrix description of astigmatic and cylindrical mirror resonators with twisted axes for laser spectroscopy,” Appl. Opt. 46, 472-482 (2007).

18. J. Reid and D. Labrie, "Second-harmonic detection with tunable diode lasers-comparison of experiment and theory," Appl. Phy. B 26, 203-210 (1981).

19. P. Kluczynski, J. Gustafsson, Åsa M. Lindberg, and O. Axner, "Wavelength modulation absorption spectrometry-an extensive scrutiny of the generation of signals," Spectrochim. Acta Part B 56, 1277-1354 (2001).

20. A. Hangauer, J. Chen, and M.-C. Amann, "Modeling of the $n$th harmonic spectra used in wavelength modulation spectroscopy and their properties," Appl. Phys. B 90, 249-254 (2008).
21. "Manual of Model SR830 DSP Lock-In Amplifier," Standford Research Systems.

22. R. Arndt, "Analytical line shapes for Lorentzian signals broadened by modulation," J. Appl. Phys. 36, 2522-2524 (1965).

23. S. Schilt, L. Thévenaz, and P. Robert, "Wavelength modulation spectroscopy: combined frequency and intensity laser modulation," Appl. Opt. 42, 6728-6738 (2003).

24. J. Chen, A. Hangauer, R. Strzoda, and M. C. Amann, "Tunable diode laser spectroscopy with optimum wavelength scanning," Appl. Phys. B 100, 331-339 (2010). DOI 10.1007/s00340-0103973-2.

25. J. Hodgkinson, D. Masiyano, and R. P. Tatam, "Gas cells for tunable diode laser absorption spectroscopy employing optical diffusers. Part 1: single and dual pass cells," Appl. Phys. B 100, 291-302 (2010).

26. W. Freude, Optische Kommunikationstechnik (SpringerVerlag, 2002), Chap. 5.

27. J. W. Goodman, "Statistical properties of laser speckle patterns," in Laser Speckle and Related Phenomena, J. C. Dainty, ed. (Springer, 1976), Chap. 2.

28. J. Chen, A. Hangauer, R. Strzoda, and M.-C. Amann, "Lowlevel and ultra-low volume hollow waveguide based carbon monoxide sensor," Opt. Lett. (to be published). 